\title{
Research Paper: Effect of Six Weeks of Neuromus- cular and Performance Balance Training on Balance and Performance Lower Extremity Climber Girls
}

\author{
Seyedeh Sara Homaye Razavi ${ }^{1}$ (D), Seyed Sadradin Shojaedin² ${ }^{*}$, Zahra Karimi ${ }^{3}$, Somayeh Barzeh $^{3}$ (D) \\ 1. Department of Pathology and Corrective Movements, Azad University, Karaj Branch, Karaj, Iran. \\ 2. Department of Corrective Exercise and Sport Injuries, School of Physical Education and Sports Sciences, International Campus, Kharazmi University \\ Tehran, Iran. \\ 3. Department of Pathology and Corrective Movements, South Tehran Azad University Branch, Tehran, Iran.
}

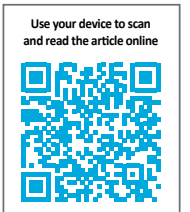

ditation Homaye Razavi SS, Shojaedin SS, Karimi Z, Barzeh S. Effect of Six Weeks of Neuromuscular and Performance Balance Training on Balance and Performance Lower Extremity Climber Girl. Physical Treatments. 2020; 10(4):213-220. http:// dx.doi.org/10.32598/ptj.10.4. 431.3

dol: : http://dx.doi.org/10.32598/ptj.10.4. 431.3

\section{(i) (5)}

Article info:

Received: 07 Nov 2019

Accepted: 13 Jul 2020

Available Online: 01 Oct 2020

Keywords:

Neuromuscular, Performance balance training, Balance, Performance, Rock climbing

\section{A B S T R A C T}

Purpose: Maintaining balance in the feet and wrists while helping to improve poor center of gravity adjustment, balancing and properly applying force to climb climbing requirements. The aim of this study was to evaluate the effect of six weeks of training on neuromuscular performance balance and lower extremity function Girls rock climbing.

Methods: Purposive sampling and available with number 30 (age $18 \pm 25$ years) were randomly divided into two experimental groups and one control group of 10 neuromuscular performances $(n=10)$ groups. Prior to the training protocol (6 weeks), to measure performance balance and lower limb of the test subjects were used Star and functional movement screening tests. Paired $\mathrm{t}$-test and analysis of covariance were used. The significant level of $\mathrm{P} \leq 0.05$ was used.

Results: The results showed that there was a significant difference in the balance and function of the lower extremities between the two neuromuscular and functional groups, but these differences were not significant between the two experimental groups.

Conclusion: The results show that the performance and functional neuromuscular training programs to improve balance and lower extremity function Girls rock climbing and lead to increased athletic performance.

* Corresponding Author:

Seyed Sadradin Shojaedin, PhD.

Address: Department of Corrective Exercise and Sport Injuries, School of Physical Education and Sports Sciences, International Campus, Kharazmi University, Tehran, Iran.

Phone: +98 (912) 5073897

E-mail:sa_shojaedin@yahoo.com 


\section{Highlights}

- Neuromuscular exercises improve balance among rock climbing girls.

- Improving balance among rock climbing girls after performing neuromuscular and functional exercises improved motor function.

- Both exercises improved lower limb function compared to the control group.

\section{Plain Language Summary}

Rock climbing is a sport that is full of excitement and the girls' interest in this field is increasing. Balancing is very important in this field, which needs to be strengthened among girl's athletes and those interested in this field. But ways to improve this factor will be very important because the choice of type of training based on criteria such as lower cost, ease of execution and its effectiveness is approved. Therefore, it is necessary to study this group of athletes. Neuromuscular training can be a good choice due to its physiological nature and effectiveness through receptors in the ankle and knee joints. Therefore, in this study, the effectiveness of neuromuscular and functional exercises on balance and lower limb function in rock climbing girls was investigated. In this study, two types of exercises were compared: functional exercises and neuromuscular exercises. Finally, both exercises were shown to improve balance and improve performance. The important point in this study was the lack of difference in effectiveness between neuromuscular and functional training; therefore, both exercises are equally effective.

\section{Introduction}

ports competitions are dangerous for all athletes. However, some sports competitions, including rock-climbing, are inherently risky [1]. Rock-climbing is related to a group of unique sports that require special and related require-

ments. Because of a long time of activity, the athlete's body muscles, especially the lower and upper limbs, remain in a state of static contraction. The arms are located on a higher level than the heart at long times of activity; therefore, these factors might decrease the available blood of active tissues, especially contracting muscles. Together, they increase the risk of membrane and tissue damage and may cause inflammatory responses [2]. The ultimate goal of various exercises has always been to improve the function and the peak of the athlete's performance, especially during competitions. For this reason, coaches have always attempted to increase the pressure of their training in this period [2].

The foot joint is among the most critical joints in the body for other limbs; accordingly, it is more prone to injury. Postural control is crucial for the optimal performance of athletic skills. The ankle joint is most likely to be injured during exercise. Functional instability refers to the foot's tendency to frequent sprains or fatigue, making a person more prone to injury [3]. Lower ex- tremity injuries, especially in the ankle, not only prevent the athlete from the sports field for a long time but also cause high medical costs [4].

An exercise that can reduce the athlete's rehabilitation period is performing neuromuscular practices. These exercises aim to improve the function of the mechanoreceptors and to increase the neuromuscular coordination, agility, and balance of the injured. Therefore, they help the person to faster return to the functional state before the injury [5]. By improving the quality of sensory nerves to deliver accurate and timely data to the Central Nervous System (CNS), neuromuscular training allows the system to activate the motor nerves of the muscles with a specific pattern and increase joint awareness and reduce organ damage [6]. Furthermore, functional training is a set of sports activities, i.e., based on the pattern of daily living activities; designed for walking, going up and down the stairs, moving the body, sitting, standing up, and moving light objects. These exercises emphasize physical fitness characteristics, such as endurance, strength, flexibility, and balance [7].

According to the principles of functional training, exercises should develop the highest possible necessary motor capabilities, skills, and preparation by performing the task, activity, or exercise. The program's focus should also be on the exercises with the most transfer. Accordingly, attention should be paid to the design of exercise 
programs, i.e., an essential part of functional training. Due to the similarity of the patterns of functional training with the main movements, these exercises have more capacity respecting sports acceptance by athletes and coaches. They are also effective in achieving the goals of injury prevention [8].

However, scholars overlooked investigating the effects of functional training and how it is used, and its possible results on the balance of rock climbers. Limited research has been conducted on the effects of functional training, i.e., a relatively novel method. Functional training is adaptable to any level of physical fitness and triggers more muscle units, compared to repetitive aerobic exercise; it results in cardiovascular endurance, strength, and flexibility $[9,10]$.

A necessary factor to reduce injury and present optimal performance in rock-climbing is to maintain proper balance in the lower extremities. Proprioception and muscle strength are essential for maintaining proper balance. Balance is an important criterion for athletes; thus, an imbalance is among the most frequent problems in this population. Besides, female athletes are more likely to be injured during exercise, compared to their male counterparts. These injuries are more prevalent in the knee and ankle [11]. Encouraging women to join exciting activities like rock-climbing on the one hand, and further research on women to clarify different aspects of this field, was among the innovations and necessities of conducting the present study. A reason for choosing two neuromuscular and functional training protocols was their similarities and differences. The similarity of these exercise types is the improvement and greater coordination of the body's motor sense system.

On the other hand, the difference between them is the principle in designing functional training that suggests the exercises should be similar to the movements and exercises of the desired sport. Moreover, previous studies reported contradictions in the effectiveness of both training types; there is no consensus among researchers on the most effective training method in this area $[8,10]$. Therefore, given the importance of improving the athletes' performance, this study aimed to investigate the effects of 6 weeks of neuromuscular and functional training on balance and lower extremity function in rockclimbing females.

\section{Materials and Methods}

This was an applied and quasi-experimental research with a pretest-posttest and a control group design. The statistical population of the present study consisted of all rock-climbing females in North Khorasan Province, Iran, with an age range of 18-25 years. In total, 30 subjects were selected using purposive and convenience sampling methods. They were randomly divided into neuromuscular training, functional training, and control groups ( $n=10 /$ group). The inclusion criteria of the study included the following: being in the age range of 18-25 years, attending regular sports and physical activity, and having physical and cardiovascular health with the approval of a specialized and committed physician.

The exclusion criteria of the study were a history of fracture in the past year; a history of specific conditions, including cardiovascular, metabolic, musculoskeletal, joint diseases, etc.; corticosteroid injection in the last 6 months, and the absence of postural abnormalities or injuries affecting the research process. The study subjects provided the informed consent form before completing the training protocol and after collecting personal information. Then, after signing the written consent and the physician's confirmation regarding the safety of the exercises, the pretest variables (dynamic balance \& lower extremity function) were measured. The dynamic balance and lower extremity function of the study subjects in the pretest stage were assessed using the star test and Functional Movement Screen (FMS) tests, respectively. Next, the research intervention groups performed neuromuscular and functional training for 6 weeks (three 45-minute sessions per week). After completing the exercises, the research variables were measured in the posttest stage. The first and last 10 minutes of each session was dedicated to warming up and cooling down.

Additionally, during the research, the study subjects' willingness to participate in the research and remaining unharmed by performing the exercises were considered. The exercises were selected according to the literature and the opinion of science experts. Besides, the control group participated in their daily exercise program throughout the study as the experimental groups; did not participate in any other training. Moreover, the control group did not perform any training protocol for the experimental groups.

In this study, the exercises of Elis et al. (2010) were used as the training protocol [12]. Exercises used in this protocol were the specific ankle joint stabilization practices, followed by exercises for maintaining the whole-body. Exercises included the following movements: walking back and forth on the balance board; single-leg stance; single-leg jump and jumping with two feet; 4) walking up and down on the sloping surface; 5 maintaining a bal- 
ance against the elastic band, and maintaining balance on one foot on the board. The exercises were performed in 6 stations, i.e., performed twice in each movement station. There was a 30-second break between each movement and a 1-minute break between each station. Each station had a maximum of 4 minutes, i.e., $<4$ minutes due to the nature of training per station. The intensity and difficulty of training increased twice during the 6 weeks of training by minor changes per station [12].

The exercise protocol of King et al. (2000) was used in this study [13]. Functional training was performed for 6 weeks and 3 sessions per week ( 75 minutes per session). Training sessions included 10 minutes of warm-up, $55 \mathrm{~min}$ utes of functional training taken from daily living activities with an emphasis on improving strength, endurance, and flexibility, and finally 10 minutes of cool-down exercises.

The functional exercise program was as follows:

1. Walking forward for 10 minutes; carrying a weight of 500 grams

\section{Sitting and standing up from a chair (5 minutes)}

3. Spiral movement between 8 cones with a one-meter distance (5 minutes)

4. Standing on toes and heels with a change in the direction of the hand to the side, front, and back (10 minutes: 40 seconds movements, 20 seconds rest)

5. Climbing the platform with a height of $20 \mathrm{~cm}$; carrying a weight of 100 grams ( 5 minutes)

6. Performing the angel balance pose with the left and right feet ( 5 minutes)

7. Dynamic stretching movements in a sitting position with weights while emphasizing strengthening the large muscles of the body (15 minutes) [13].
Star balance test was used to measure dynamic equilibrium. In this test, a star with 8 directions is drawn on the ground, and the subject puts her/his upper leg in the center of the star and tries to reach the 8 directions with the other leg. If the right foot is dominant, the test is taken counterclockwise; otherwise, the test is conducted clockwise. The distance from the point of contact of the free foot to the center of the star is the reaching distance. Each subject performs each direction three times. Then, the average value is calculated and divided by the length of the leg in centimeters. Next, the obtained number is multiplied by 100 to obtain the reaching distance in terms of a percentage of the size of the leg length [14].

The FMS test set was designed to simultaneously assess mobility and stability using 7 motion tests. These tests can be performed in 5 to 10 minutes; therefore, they can easily be used by coaches for pre-season assessments. In this study, FMS-7 tests were used, including the deep squat, stepping over obstacles, lunge, shoulder mobility, active straight leg raise, trunk stabilization swim, and rotational stability [15].

Initially, the normality of data distribution was investigated by the Shapiro-Wilk test. Besides, the mean and standard deviation scores were used to describe the collected data. Then, the analysis of covariance (ANCOVA) and Least Significant Difference (LSD) test were used to evaluate the obtained results in SPSS. The significance level was set at $\mathrm{P} \leq 0.05$.

\section{Results}

Table 1 presents the group-wise demographic characteristics of the study subjects. The ANCOVA data suggested a significant difference in balance between the control and neuromuscular groups $(\mathrm{F}=88.11, \mathrm{P}=0.003)$ as well as the control and functional groups $(\mathrm{F}=20.61$, $\mathrm{P}=0.001$ ) (Figure 1). The ANCOVA results also indicated that lower extremity function was significantly different between the control and neuromuscular groups $(\mathrm{F}=26.43$, $\mathrm{P}=0.001$ ) as well as the control and functional groups

Table 1. The anthropometric characteristics of the research groups

\begin{tabular}{|c|c|c|c|c|c|}
\hline \multirow{2}{*}{ Group } & \multicolumn{4}{|c|}{ Mean $\pm S D$} & \multirow{2}{*}{$\mathbf{P}$} \\
\hline & Age (y) & Height $(\mathrm{cm})$ & Weight (kg) & BMI $\left(\mathrm{kg} / \mathrm{m}^{2}\right)$ & \\
\hline Control & $22.34 \pm 4.31$ & $174.10 \pm 7.21$ & $69.80 \pm 7.3$ & $23.02 \pm 7.25$ & 0.934 \\
\hline Neuromuscular & $19.21 \pm 2.11$ & $170.20 \pm 5.96$ & $70.12 \pm 6.12$ & $24.20 \pm 5.04$ & 0.514 \\
\hline Functional & $21.12 \pm 2.22$ & $168.40 \pm 6.20$ & $66.40 \pm 10.21$ & $23.41 \pm 7.20$ & 0.713 \\
\hline
\end{tabular}




\section{Balance}

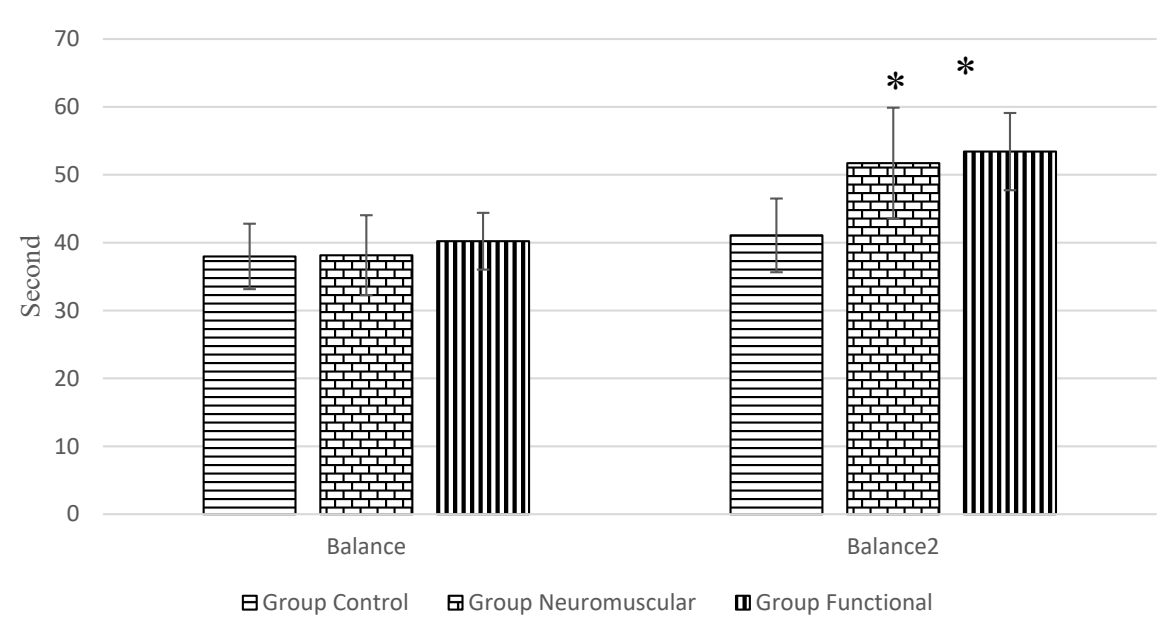

Figure 1. Comparing balance changes in the research groups

PHYSICAL TREA TMENTS

*Significant differences comparisons concerning the control group and the pretest stage $(\mathrm{P}<0.05)$

Balance: pretest stage balance; Balance 2: posttest stage balance (inter-group differences)

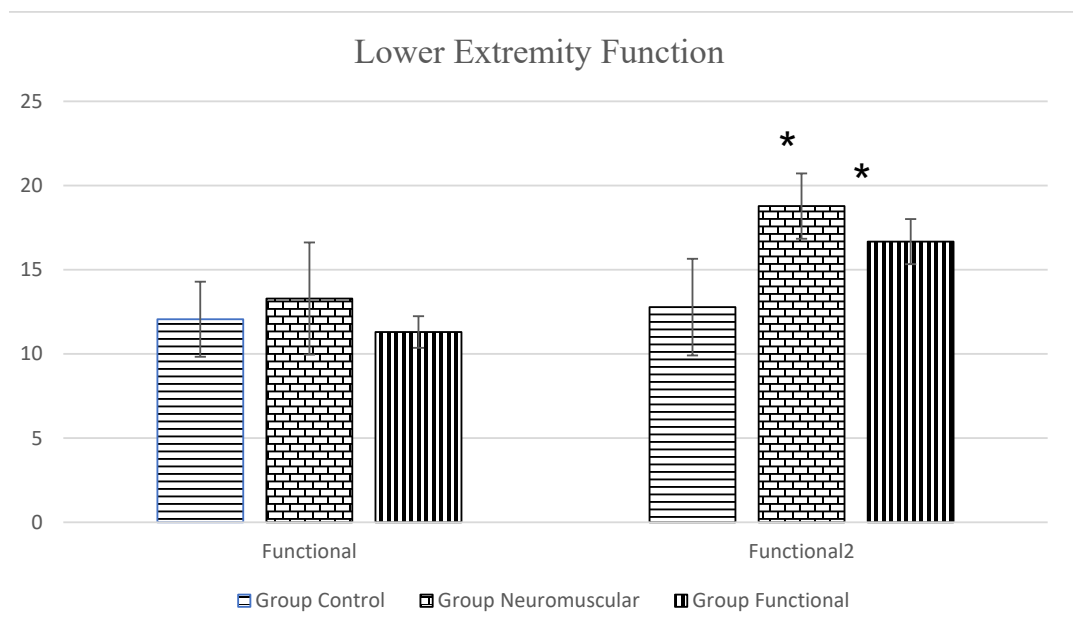

Figure 2. Comparing lower extremity function changes in the research groups

PHYSICAL TREA $\mid$ MENTS

*Significant difference comparisons concerning the control group and pretest stage $(P<0.05)$

Functional group: Lower-extremity function in the pretest stage; Functional 2: lower extremity function in the posttest stage (inter-group differences).

$(\mathrm{F}=40.38, \mathrm{P}=0.001$ ) (Figure 2). Furthermore, ANCOVA data revealed that balance $(\mathrm{P}=0.7)$ and lower extremity function $(\mathrm{P}=0.1)$ were significantly different between the functional and neuromuscular groups $(\mathrm{P}>0.05)$.

Moreover, there existed significant intra-group differences in balance $(\mathrm{P}=0.001)$ and lower extremity function $(\mathrm{P}=0.001)$ in the neuromuscular group as well as balance $(\mathrm{P}=0.001)$ and lower extremity function $(\mathrm{P}=0.001)$ in the functional exercise group. The frequency of balance changes was $26.23 \%$ and $24.69 \%$ in the neuromuscular and functional groups, respectively. Besides, the frequency of lower extremity function alternations was
$29.22 \%$ and $32.24 \%$ in the neuromuscular and functional groups, respectively.

The ANCOVA data provided in Table 1 reflected that the distribution of demographic data in all three groups (neuromuscular, functional, \& control) was normal.

\section{Discussion}

The rock-climbing sport requires high strength, especially in the shoulder girdle, pelvic girdle, and trunk muscles. Excessive work on one part of the body can lead to injury [2]. Therefore, it might play an important 
role in preventing injury to some extent by observing preventive measures. Therefore, this study aimed to investigate the effects of 6 weeks of neuromuscular training on balance and lower extremity function in rock-climbing females. The results suggested a significant difference in the experimental groups respecting pretest-posttest balance and lower extremity function scores. These exercise types significantly increased the balance and function of the lower extremity in the explored subjects.

Besides, in this regard, the most significant effect was related to the neuromuscular training group. However, no significant difference was observed between the experimental groups. There was also no significant difference in the control group for both research variables. The obtained results about the effectiveness of exercises on improving balance were consistent with those of Vitale et al. [16], Bouteraa et al. [17], and Hopper and et al. [18]. However, the present study data were inconsistent with those of Whitacre and associates [19]. Possible reasons for this inconsistency can be explained by a difference in the duration of the training courses of the mentioned research and the present study; or it can be attributed to differences in the nature of the training protocol, as well as the different tools used in these investigations.

Rozzi et al. (1999) reported that performing 4 weeks of balance training in individuals with functional ankle instability significantly increased the score of the ankle function assessment tool, compared to the healthy controls [20].

A necessary factor to reduce injury and achieve optimal performance in rock-climbing is to maintain proper balance in the lower extremities. Proprioception and muscle strength are essential for maintaining proper balance. Balance is a crucial criterion for athletes; thus, an imbalance is among the most common problems they encounter. There are various methods to prevent this prevalent injury, not only in healthy athletes but also in athletes with functional and biomechanical instability [21]. Explaining the causes and mechanisms that justify improving balance requires referring to the various components of the sensory-motor system responsible for maintaining balance. This system includes the sensory, motor, and central processing components.

Furthermore, balance-related neuromuscular training requires motor control responses at the level of the brainstem. Movement control can be improved at all levels using neuromuscular exercises. Movement control is a crucial principle of balance rehabilitation and proprioception. This is because proper movement control requires reflex responses at the level of the spinal cord, postural reactions, as well as spontaneous balance at the level of the brainstem, and conscious responses at the level of the cortex [20].

The significance of the effect of functional training in the present study can be attributed to several factors, as follows: balance is a multifactorial issue, i.e., affected by various factors, such as strength, proprioception, reaction speed, and motor coordination [21]. As individuals age, their muscle strength, proprioception information quality, and rate of reaction are affected and disrupted. Functional training can simultaneously develop and improve the above-mentioned characteristics [22]. It has also been suggested that this exercise technique can increase mobility and joint stability in all motor plates, and improve balance [23].

Exercises for the torso and muscles of the central area improve balance, vertical jumping, jumping, and Cooper tests' values. We are likely in dire need of endurance, agility, and balance in rock-climbing; these activities are considered essential in rock-climbing. Trunk muscles are classified locally and generally, depending on the function and anatomical angle. Local muscles with the most direct and indirect connections to the fixed parts of the lumbar vertebrae and general muscles are connected to the thighs and pelvis; they produce torque and load transfer between the chest and pelvis, and ultimately help maintain balance.

Therefore, coordination and contraction in general and local muscles are essential in maintaining balance and function. It also reduces the risk of lower extremity injuries. Therefore, athletes show interest in performing trunk exercises as an exercise program to improve lower extremity and prevent back injuries [24]. A possible mechanism for improving individuals' balance (which results from functional training), is that functional training uses leg, wrist, knee, and thigh muscles to apply natural pressure on all joints within the motor chain. It seems to be highly beneficial for improving the performance of the proprioception.

Furthermore, functional activities coordinate proprioception feedback sent from Pacinian Corpuscles, Ruffini Endings, Golgi-Mazzoni Corpuscles, and Golgi tendon organs with the practical use of multi-joint and multi-faceted movements. Due to this process and increasing the functional coordination, it develops balance and function in the lower extremity of rock-climbing girls [25]. The ability of individuals to maintain optimal motor function and control posture is essential for the successful performance of almost all daily movements. According to the systems the- 
ory, the ability to control the position of the body in space due to interaction results from the simultaneous and complex effects of the nervous, muscular, and skeletal systems, i.e., collectively called the postural control system [26].

This system requires the integration of sensory data to control the position to maintain balance and subsequent movement, to detect the body position in space, as well as the ability of the musculoskeletal system to exert force. According to this theory, musculoskeletal factors affecting balance regulation include the muscle characteristics, joint motion range, and biomechanical connection of different parts of the body. The NS also significantly affects controlling the condition.

A possible mechanism of the further significant effect of neuromuscular training, compared to functional training was the increase in the attention in neuromuscular training. Attention is a neuropsychological process through which the CNS affects the received data. Neuromuscular training might increase attention to the symptoms of proprioception by the brain; first on a conscious level, then on an unconscious level after the exercise. This process can activate pathways, increase the number of synapses, and enhance the corresponding sensory region observed in plasticity. Therefore, according to the cases mentioned, neuromuscular training can increase balance and lower extremity function in rock-climbing girls.

We observed significant effects of neuromuscular and functional training types on balance and lower extremity function in rock-climbing girls. Besides, the superiority of neuromuscular training in these variables over functional training was detected. Accordingly, the following points are suggested: the evaluation of the durability of the exercises used in the present study on rock-climbing athletes and other sports; it is recommended to conduct similar research on men with different age ranges and compare it with the present study results, and it is recommended to perform similar research using more accurate laboratory measurement tools. Besides, the lack of access to larger sample size and the lack of financial and laboratory resources to examine the indicators through kinematic systems were among the limitations of the present study.

\section{Conclusion}

The present study results indicated that neuromuscular and functional training programs increase balance and lower extremity function in rock-climbing girls, followed by improved athletic performance. However, in this study, the effectiveness of neuromuscular train- ing was higher than that of the functional training on the above-mentioned factors.

The study examined the effect of each intervention (neuromuscular \& functional) to compare the effects of neuromuscular training types on balance and lower extremity function in rock-climbing girls. There was no significant difference regarding balance and lower extremity function in the neuromuscular and functional training groups, compared to the controls. Therefore, although neuromuscular and functional training improved balance and lower extremity function in rock-climbing girls, the effectiveness of neuromuscular training was slightly higher. However, this increase was not statistically significant.

\section{Ethical Considerations}

\section{Compliance with ethical guidelines}

There were no ethical considerations to be considered in this research.

\section{Funding}

The study was extracted from Master's thesis of a Corrective Movement and Sports Injury student affiliated to Karaj Azad University.

\section{Authors' contributions}

Investigation, Methodology: Seyedeh Sara Hooma Razavi, Seyed Sadradin Shojaedin, and Zahra Karimi Somayeh Barzeh; Writing - original draft, and Writing - review \& editing: All authors.

\section{Conflict of interest}

The authors declared no conflict of interest.

\section{References}

[1] Gasemi B, Azamian Jazi A, Noori P. [The effect of 12 weeks functional training on dynamic balance in healthy older women (Persian)]. Salmand: Iranian Journal of Ageing. 2011; 5(4):30-6. http:/ / salmandj.uswr.ac.ir/article-1-263-en.html

[2] Khajeie R, Asghari E, Arazi H, Kari M, Seyyed Ahmadi M. [Description of the changes of some humoral immune variables immediately and 24 hours after exercise during the preparation exercises for rock climbing (Persian)]. Journal of Sabzevar University of Medical Sciences. 2012; 19(2):136-45. http://jsums.medsab.ac.ir/article_280_0.html 
[3] Holmes A, Delahunt E. Treatment of common deficits associated with chronic ankle instability. Sports Medicine. 2009; 39(3):207-24. [DOI:10.2165/00007256-200939030-00003] [PMID]

[4] McGuine TA, Keene JS. The effect of a balance training program on the risk of ankle sprains in high school athletes. The American Journal of Sports Medicine. 2006; 34(7):1103-11. [DOI:10.1177/0363546505284191] [PMID]

[5] Soligard T, Myklebust G, Steffen K, Holme I, Silvers H, Bizzini M, et al. Comprehensive warm-up programme to prevent injuries in young female footballers: Cluster randomised controlled trial. BMJ. 2008; 337:a2469. [DOI:10.1136/bmj. a2469] [PMID] [PMCID]

[6] de Vries JS, Krips R, Sierevelt IN, Blankevoort L, Van Dijk CN. Interventions for treating chronic ankle instability. Cochrane Database of Systematic Reviews. 2011; (8):CD004124. [DOI:10.1002/14651858.CD004124.pub3] [PMID]

[7] Heinrich K, Becker C, Carlisle T, Gilmore K, Hauser J, Frye J, et al. High-intensity functional training improves functional movement and body composition among cancer survivors: A pilot study. European Journal of Cancer Care. 2015; 24(6):812-817. [DOI:10.1111/ecc.12338] [PMID]

[8] Steffen K, Bakka HM, Myklebust G, Bahr R. Performance aspects of an injury prevention program: A ten-week intervention in adolescent female football players. Scandinavian Journal of Medicine \& Science in Sports. 2008; 18(5):596-604. [DOI:10.1111/j.1600-0838.2007.00708.x] [PMID]

[9] Heinrich KM, Becker C, Carlisle T, Gilmore K, Hauser J, Frye J, et al. High-intensity functional training improves functional movement and body composition among cancer survivors: A pilot study. European Journal of Cancer Care. 2015; 24(6):812-7. [DOI:10.1111/ecc.12338] [PMID]

[10] Murawska-Cialowicz E, Wojna J, Zuwala-Jagiello J. Crossfit training changes brain-derived neurotrophic factor and irisin levels at rest, after wingate and progressive tests, and improves aerobic capacity and body composition of young physically active men and women. Journal of Physiology and Pharmacology: An Official Journal of the Polish Physiological Society. 2015; 66(6):811-21. [PMID]

[11] Räisänen AM, Kulmala T, Parkkari J, Vasankari T, Kannus $\mathrm{P}$, Krosshaug T, et al. There is no relationship between lower extremity alignment during unilateral and bilateral drop jumps and the risk of knee or ankle injury: A prospective study. Journal of Orthopaedic \& Sports Physical Therapy. 2020; 50(5):267-74. [DOI:10.2519/jospt.2020.9247] [PMID]

[12] Eils E, Schröter R, Schröder M, Gerss J, Rosenbaum D. Multistation proprioceptive exercise program prevents ankle injuries in basketball. Medicine \& Science in Sports \& Exercise. 2010; 42(11):2098-105. [DOI:10.1249/ MSS.0b013e3181e03667] [PMID]

[13] King MB, Judge JO, Whipple R, Wolfson L. Reliability and responsiveness of two physical performance measures examined in the context of a functional training intervention. Physical Therapy. 2000; 80(1):8-16. [DOI:10.1093/ptj/80.1.8] [PMID]

[14] Bashiri J, Hadi H, Bashiri M, Rostamkhany H. [Comparison effect of six week resistance-balance, velocity-balance and balance training on dynamic balance in active elderly males (Persian)]. Journal of Research in Rehabilitation Sciences. 2009; 5(2):104-15. [DOI: 10.22122/jrrs.v5i2.110]
[15] Brown P. Movement: Functional movement systemsscreening, assessing, corrective strategies on target publications. The Journal of the Canadian Chiropractic Association. 2012; 56(4):316. https://www.ncbi.nlm.nih.gov/pmc/articles/PMC3501919/

[16] Vitale JA, La Torre A, Banfi G, Bonato M. Effects of an 8-week body-weight neuromuscular training on dynamic balance and vertical jump performances in elite junior skiing athletes: A randomized controlled trial. The Journal of Strength \& Conditioning Research. 2018; 32(4):911-20. [DOI:10.1519/JSC.0000000000002478] [PMID]

[17] Bouteraa I, Negra Y, Shephard RJ, Chelly MS. Effects of combined balance and plyometric training on athletic performance in female basketball players. Journal of Strength and Conditioning Research. 2020; 34(7):1967-73. [DOI:10.1519/ JSC.0000000000002546] [PMID]

[18] Hopper A, Haff EE, Barley OR, Joyce C, Lloyd RS, Haff GG. Neuromuscular training improves movement competency and physical performance measures in 11-13-yearold female netball athletes. The Journal of Strength \& Conditioning Research. 2017; 31(5):1165-76. [DOI:10.1519/ JSC.0000000000001794] [PMID]

[19] Whitacre TD, Toland CE, Clark KP, editors. Effects of a short-term neuromuscular training program on jump performance and landing mechanics. Annual scientific meeting, 2017 november $4^{\text {th }} 5^{\text {th }}$, San Diego, CA. International Journal of Exercise Science. 2018; 6(9). https://digitalcommons.wku.edu/cgi/viewcontent.cgi?article $=3641 \&$ context =ijesabhttps://digitalcommons.wku.edu/cgi/viewcontent. cgi?article $=3641 \&$ context $=$ ijesab

[20] Rozzi SL, Lephart SM, Sterner R, Kuligowski L. Balance training for persons with functionally unstable ankles. Journal of Orthopaedic \& Sports Physical Therapy. 1999; 29(8):478-86. [DOI:10.2519/jospt.1999.29.8.478] [PMID]

[21] Behm D, Colado JC. The effectiveness of resistance training using unstable surfaces and devices for rehabilitation. International Journal of Sports Physical Therapy. 2012; 7(2):22641. [PMCID] [PMID]

[22] Farsi A, Abdoli B, Baraz P. [Effect of balance, strength, and combined training on the balance of the elderly women (Persian)]. Salmand: Iranian Journal of Ageing. 2015; 10(3):54-61. http:/ / salmandj.uswr.ac.ir/article-1-726-en.html

[23] Gaedtke A, Morat T. TRX suspension training: A new functional training approach for older adults-development, training control and feasibility. International Journal of Exercise Science. 2015; 8(3):224-33. [PMCID]

[24] Kosmata A. Functional exercise training with the trx suspension trainer in a dysfunctional, elderly population [MSc. Thesis]. United States: Appalachian State University; 2014. https:/ / libres.uncg.edu/ir/asu/listing.aspx?id=17069

[25] Imai A, Kaneoka K, Okubo Y, Shiraki H. Effects of two types of trunk exercises on balance and athletic performance in youth soccer players. International Journal of Sports Physical Therapy. 2014; 9(1):47. [PMID] [PMCID]

[26] Lazarus NR, Harridge SDR. Declining performance of master athletes: Silhouettes of the trajectory of healthy human ageing? The Journal of Physiology. 2017; 595(9):2941-8. [DOI:10.1113/JP272443] [PMID] [PMCID] 